\title{
Author Correction: Single-molecule imaging by optical absorption
}

Michele Celebrano, Philipp Kukura, Alois Renn and Vahid Sandoghdar

Correction to: Nature Photonics https://doi.org/10.1038/nphoton.2010.290, published online 9 January 2011.

In the Supplementary Video initially published with this Letter, the right-hand panel displaying the fluorescence emission was not showing on some video players due to a formatting problem; this has now been fixed. The video has also now been amended to include colour scale bars for both the left- (differential transmission signal) and right-hand panels.

Published online: 12 April 2018

https://doi.org/10.1038/s41566-018-0159-7 